\title{
A deep-learning based native-language classification by using a latent semantic analysis for the NLI Shared Task 2017
}

\author{
Yoo Rhee Oh, Hyung-Bae Jeon *, Hwa Jeon Song, \\ Yun-Kyung Lee, Jeon-Gue Park, and Yun-Keun Lee \\ Speech Intelligence Research Group, \\ Electronics and Telecommunications Research Institute, South Korea \\ \{yroh, hbjeon, songhj, yunklee, jgp, yklee\} @etri.re.kr
}

\begin{abstract}
This paper proposes a deep-learning based native-language identification (NLI) using a latent semantic analysis (LSA) as a participant (ETRI-SLP) of the NLI Shared Task 2017 (Malmasi et al., 2017) where the NLI Shared Task 2017 aims to detect the native language of an essay or speech response of a standardized assessment of English proficiency for academic purposes. To this end, we use the six unit forms of a text data such as character 4/5/6-grams and word 1/2/3-grams. For each unit form of text data, we convert it into a count-based vector, extract a 2000-rank LSA feature, and perform a linear discriminant analysis (LDA) based dimension reduction. From the count-based vector or the LSA-LDA feature, we also obtain the output prediction values of a support vector machine (SVM) based classifier, the output prediction values of a deep neural network (DNN) based classifier, and the bottleneck values of a DNN based classifier. In order to incorporate the various kinds of text-based features and a speech-based i-vector feature, we design two DNN based ensemble classifiers for late fusion and early fusion, respectively. From the NLI experiments, the F1 (macro) scores are obtained as 0.8601, 0.8664 , and 0.9220 for the essay track, the speech track, and the fusion track, respectively. The proposed method has comparable performance to the top-ranked teams for the speech and fusion tracks, although it has slightly lower performance for the essay track.
\end{abstract}

${ }^{*}$ Corresponding author

\section{Introduction}

Native-language identification (NLI) can be used to improve the performance of automatic speech recognition (ASR) for non-native speakers using native-language (L1) specific ASR systems. NLI can also be used in a computer-assisted language learning system using the L1-specific target-language errors. A considerable body of research on NLI has been reported (Malmasi, 2016; Malmasi and Dras, 2015) and the developed approaches can be classified into text-based NLI (Tetreault et al., 2013), speech-based NLI (Malmasi et al., 2016), and text and speech based NLI (Zampieri et al., 2017). Among them, this paper focuses on the NLI of text and speech data for the NLI Shared Task 2017 (Malmasi et al., 2017).

The first NLI Shared Task aims to identify the L1 of the text data of an essay response (Tetreault et al., 2013). Notably, a part of the 2016 Computational Paralinguistics Challenge focuses on speech-based NLI (Schuller et al., 2016). This year, the goal of the NLI Shared Task 2017 is to detect the L1 of the essay and speech responses of a standardized assessment of English proficiency for academic purposes among eleven L1s, Arabic, Chinese, French, German, Hindi, Italian, Japanese, Korean, Spanish, Telugu, and Turkish. To this end, there are 11,000 training data set, 1,100 development data set, and 1,100 test data set. In addition, each data set contains the text of an essay response, the transcription text and 800dimensional i-vector feature of a speech response, and the L1 annotation of the participant of essay and speech responses.

In this paper, we propose a deep-learning based NLI method using a latent semantic analysis (LSA) as a participant (ETRI-SLP) of the NLI Shared Task 2017. First, the higher-rank of an LSA feature is used to detect L1 information; the 
lower-rank of an LSA feature is used to detect document topic information (Jeon and Lee, 2016b; Bellegarda, 2000). Second, we adopt a state-ofthe-art machine learning methods, a deep-learning method (Jeon and Lee, 2016a; Chung and Park, in review), for L1 classification using various kinds of text-based features and a speech-based feature.

\section{Feature extraction of the proposed method}

\subsection{Data preparation}

For the text data of the NLI Shared Task 2017 such as the text of an essay response and the transcription text of a speech response, we use six unit forms for each text: (a) word 1-gram, (b) word 2-gram, (c) word 3-gram, (d) character 4-gram, (e) character 5-gram, and (f) character 6-gram. It is assumed that a word $n$-gram could reveal L1-specific words (e.g. 'kimchi' is a Korean food name) and L1-specific word sequences while a character $n$-gram could capture L1-specific typing errors, L1-specific character sequence patterns, etc.

First, each unit of a text is converted into a count-based vector and then entropy normalization (Jeon and Lee, 2016b; Bellegarda, 2000) is applied to the count-based vector. Next, the normalized count-based vector ( $\left.R a w^{\text {count }}\right)$ is used to extract the 2000-rank features of a latent semantic analysis (LSA) (Jeon and Lee, 2016b; Bellegarda, 2000). The LSA feature is subsequently compressed into 10-dimensional features using a linear discriminant analysis (LDA), which is referred to as $\operatorname{Raw}^{L S A 2000 / L D A 10}$ hereafter. It is assumed that the high-rank LSA features could capture the $\mathrm{L} 1$ characteristics.

For a speech data set of the NLI Shared Task 2017, we only use the 800-dimensional i-vector feature of each speech response, which is supported by the organizers (Malmasi et al., 2017). In addition, we apply LDA normalization to the i-vector features.

\subsection{Feature extraction}

We extract five kinds of features from the $R a w^{\text {count }}$ or $R_{a w^{L S A 2000 / L D A 10}}$ of each unit form of a text for an L1 classification: (a) the output prediction values ( $S V M_{\text {output }}^{\text {count }}$ ) of a SVM classifier using the Raw count, (b) the output prediction values $\left(S V M_{\text {output }}^{L S A 2000 / L D A 10}\right)$ of a SVM classifier using the $\operatorname{Raw}^{L S A 2000 / L D A 10}$, (c) the out-

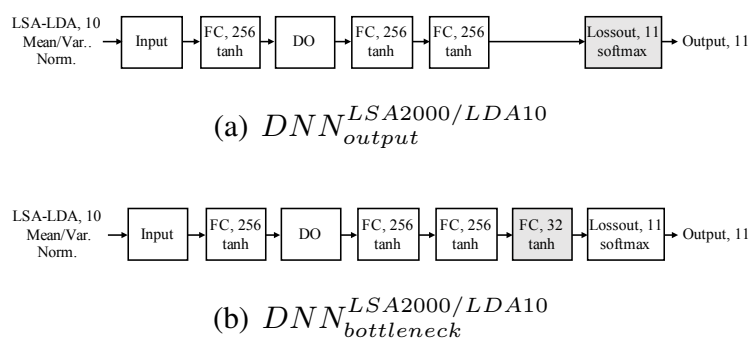

Figure 1: Configuration of the two DNNs for the $D N N_{\text {output }}^{L S A 2000 / L D A 10}$ and the $D N N_{\text {bottleneck }}^{L S A 2000 / L D A 10}$, respectively.

put prediction values $\left(D N N_{\text {output }}^{L S A 2000 / L D A 10}\right)$ of a DNN classifier using the $R a w^{L S A 2000 / L D A 10}$, (d) the bottleneck (Grézl et al., 2007) values $\left(D N N_{\text {bottleneck }}^{L S A 2000 / L D A 10}\right)$ of the last hidden layer of a DNN classifier using the $R a w^{L S A 2000 / L D A 10}$, and (e) the $R_{a w}{ }^{L S A 2000 / L D A 10}$ itself.

For the $S V M_{\text {output }}^{\text {count }}$ and $S V M_{\text {output }}^{L S A 2000 / L D A 10,}$ a linear kernel SVM is trained using SVM-Light tool (Joachims, 1999). In addition, two kinds of DNNs are trained for the $D N N_{\text {output }}^{L S A 2000 / L D A 10}$ and $D N N_{\text {bottleneck }}^{L S A 2000 / L D A 10}$, respectively, as shown in Fig. 1. In other words, the input features are normalized to a zero mean and unit variance and the output layer of each DNN is a softmax layer with eleven nodes that correspond to the eleven L1s. In order to prevent overfitting, dropout (DO) hidden layers are inserted. Moreover, each fullyconnected (FC) hidden layer uses a hyperbolic tangent (tanh) activation function. As shown in Fig. 1(a), DN $N_{\text {output }}^{L S A 2000 / L D A 10}$ consists of one input layer, four hidden layers, and one output layer. The first, third, and fourth hidden layers are FC layers where each layer contains 256 nodes, while the second hidden layer is a DO layer. On the other hand, the difference between $D N N_{\text {bottleneck }}^{L S D A 10}$ from $D N N_{\text {output }}^{L S A 2000 / L D A 10}$ is that one additional hidden layer with 32 nodes is inserted before the output layer for bottleneck feature extraction, as shown in Fig. 1(b).

\section{DNN based classifier for the NLI Shared Task 2017}

For each text of the essay response and speech response transcription, thirty kinds of features are extracted by combining the six unit forms with the five feature types. Moreover, an 800-dimensional $\mathrm{i}$-vector is extracted for each speech response signal. In order to combine the various features for 


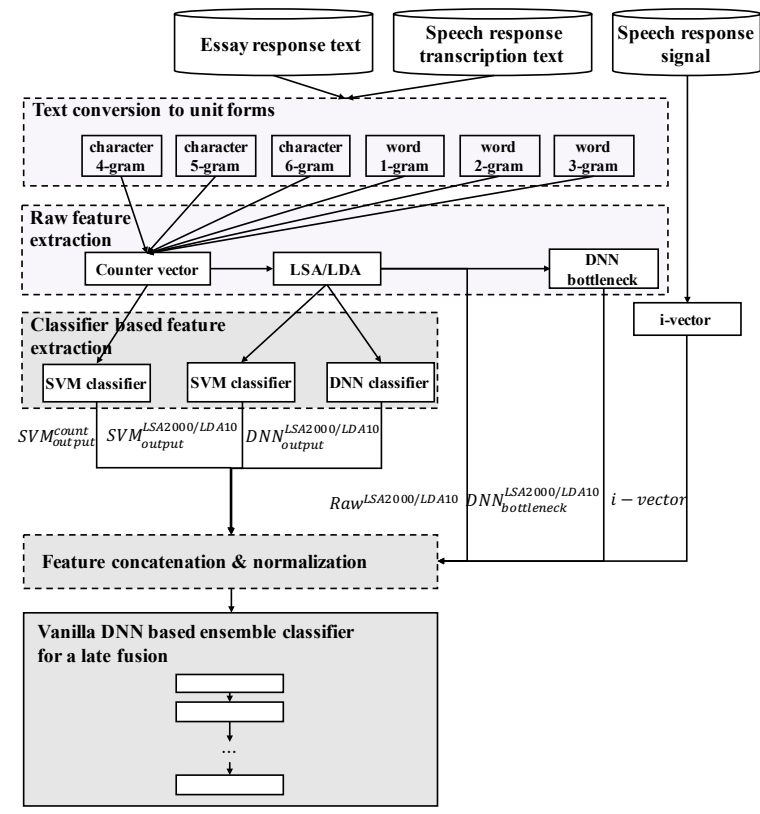

(a) A vanilla DNN based ensemble classifier for late fusion

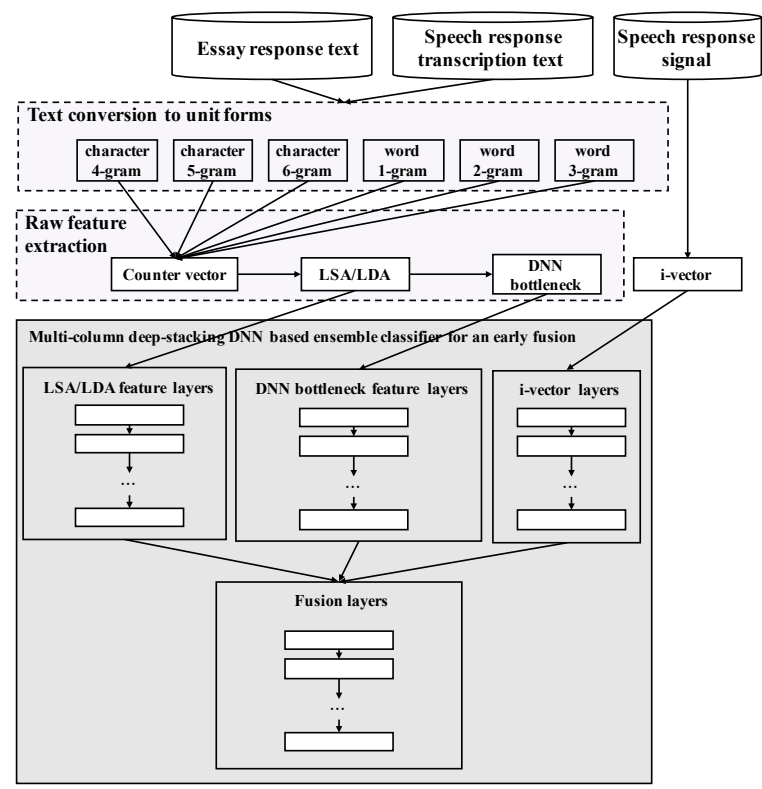

(b) A multi-column deep-stacking DNN based ensemble classifier for early fusion

Figure 2: The two kinds of DNN based ensemble classifiers for early fusion and late fusion, respectively.

the NLI Shared Task 2017, we design two DNN based classifiers: (a) a vanilla DNN based ensemble classifier for late fusion and (b) a multi-column deep-stacking DNN based ensemble classifier for early fusion, as shown in Fig. 2. Basically, each output layer of the proposed DNN based ensemble classifiers is a softmax layer with eleven nodes that correspond to the eleven native languages.
- A vanilla DNN based ensemble classifier for late fusion:

A late fusion method (Snoek et al., 2005) is a feature combination method that generates a feature-based classifier corresponding to each feature and then performs classification using the output values of the featurebased classifiers. As shown in Fig. 2(a), the vanilla DNN based ensemble classifier is designed for late fusion using the output prediction values of the feature-based classifiers, $S V M_{\text {output }}^{\text {count }}, S V M_{\text {output }}^{L S A 2000 / L D A 10}$, and $D N N_{\text {output }}^{L S A 200 / L D A 10}$. In other words, we concatenate the text-based and speech-based features including the output values of the feature-based classifiers and then apply the concatenated feature input data of the vanilla DNN based ensemble classifier for the fusion. Moreover, the vanilla DNN based ensemble classifier consists of one input layer, several hidden layers, and one output layer.

\section{- A multi-column deep-stacking DNN based ensemble classifier for early fusion:}

An early fusion method (Snoek et al., 2005) is a feature combination method that fuses several kinds of features. As shown in Fig. 2(b), the multi-column (Ciresan et al., 2012) deep-stacking DNN based ensemble classifier is designed for early fusion. In other words, each feature is fed into the multicolumn deep-stacking DNN and then linked to the corresponding feature layer. The node values of the last hidden layer of each featurerelated layers are then connected to the input layer of the fusion-related layers. Moreover, the feature-related layers and fusionrelated layers all have different configurations since the proposed multi-column deepstacking DNN based ensemble classifier aims to efficiently combine heterogeneous features.

In particular, the overall network of the multicolumn deep-stacking DNN based ensemble classifier is trained with a single objective function while the vanilla DNN based ensemble classifier is trained with multiple object functions such as (a) the objective functions for the feature-based classifiers and (b) the objective function for fusion. In this paper, $S V M_{\text {output }}^{\text {count }}, S V M_{\text {output }}^{L S A 2000 / L D A 10}$, and $D N N_{\text {output }}^{L S A 2000 / L D A 10}$ are used as feature- 


Input, 450 $\rightarrow$ DO $\rightarrow \begin{gathered}\mathrm{FC}, 256 \\ \text { tanh }\end{gathered} \rightarrow$ DO $\rightarrow \begin{gathered}\mathrm{FC}, 256 \\ \text { tanh }\end{gathered} \rightarrow \begin{gathered}\text { Lossout, } 11 \\ \text { softmax }\end{gathered} \rightarrow$ Output, 11

Figure 3: Configuration of the vanilla DNN based ensemble classifier for the essay track, where each block indicates a layer of the DNN and the number in a block indicates the number of nodes in the corresponding layer.

based classifiers for the vanilla DNN based ensemble classifier.

\section{Results}

This section presents the submitted experimental setups and the performances for the three tracks of the NLI Shared Task 2017: (a) the essay track using the texts of the essay responses, (b) the speech track using the transcription texts and i-vector features of the speech responses, and (c) the fusion track using both the texts of the essay responses and the transcription texts and i-vector features of the speech responses. In the experiments of the essay track, we also examine the performance of each unit form of a text while the feature combinations are examined in the experiments of the speech track. In addition, the performance is compared with the classification accuracy metric when evaluating the 1,100 development data set.

\subsection{The experimental setup and its performances for the essay track}

For the L1 detection of the essay track, we only used the vanilla DNN based ensemble classifier with the assumption that the text-related features were not extremely heterogeneous for each other. The submitted ETRI-SPL NLI system for the essay track was performed as follows.

We first transformed each text data into the six unit forms such as word 1/2/3-grams and character 4/5/6-grams. Then, we extracted the five features $\left(S V M_{\text {output }}^{\text {count }}, S V M_{\text {output }}^{L S A 2000 / L D A 10}\right.$, $D N N_{\text {output }}^{L S A 2000 / L D A 10}, \quad D N N_{\text {bottleneck }}^{L S A 2000 / L D A 10}$, and $R a w^{L S A 2000 / L D A 10}$ ) for each unittransformed text. As a result, we obtained the thirty features for each text and then concatenated them into one 450-dimensional feature. The concatenated feature was then normalized to a zero mean and unit variance. After that, the normalized feature was fed into the input layer of a vanilla DNN based ensemble classifier. As shown in Fig. 3, the vanilla DNN based ensemble classifier for the essay track consisted of an input

\begin{tabular}{|c|c|c|c|}
\hline Unit & $\begin{array}{l}\text { Feature } \\
\text { dimension }\end{array}$ & $\begin{array}{l}\text { Norm. } \\
\text { method }\end{array}$ & Accuracy \\
\hline Official baseline & & & 0.7236 \\
\hline word 1-gram Raw ${ }^{L S A 2000 / L D A 10}$ & 10 & Mean/Var.. & 0.7764 \\
\hline word 2-gram Raw ${ }^{L S A 2000 / L D A 10}$ & 10 & Mean/Var. & 0.7909 \\
\hline word 3-gram Raw ${ }^{L S A 2000 / L D A 10}$ & 10 & Mean/Var. & 0.7045 \\
\hline character 4-gram Raw ${ }^{L S A 2000 / L D A 10}$ & 10 & Mean/Var. & 0.7736 \\
\hline character 5-gram Raw ${ }^{L S A 2000 / L D A 10}$ & 10 & Mean/Var. & 0.8064 \\
\hline character 6-gram $\operatorname{Raw}^{L S A 2000 / L D A 10}$ & 10 & Mean/Var. & 0.8164 \\
\hline
\end{tabular}

Table 1: Performance comparison of each unit form of the $D N N_{\text {output }}^{L S A 2000 / L D A 10}$ of the proposed method for the essay track when evaluating the development data, where 'Mean/Var.' indicates the normalization to the zero mean and unit variance.

layer, first and third DO hidden layers, second and fourth FC hidden layers, and an output layer. Each FC layer contained 256 nodes with a tanh activation function.

Prior to the performance comparison of the proposed ETRI-SPL NLI for the essay track, we evaluated the performance corresponding to each unit form. To this end, we extracted the six $\operatorname{Raw}^{L S A 2000 / L D A 10}$ features for the word $1 / 2 / 3$ grams and character 4/5/6-grams, respectively. Then, we generated the vanilla DNN based ensemble classifier using each of the six features. After that, the six classifiers were evaluated for the development data. It was shown from the second, third, and fourth rows of Table 1 that the performances corresponding to the word $n$-grams were improved except for the word 3-gram when compared to the performance of the official baseline. It was noted that the performance degradation corresponding to the word 3-gram was occurred due to a data sparseness. Moreover, it was shown from the fifth, sixth, and seventh rows of the table that the performances corresponding to the character $n$-grams were improved according to the increase of the $n$-gram order. Especially, the performance corresponding to the character 6-gram outperformed among the others.

Next, we evaluated the performance corresponding to each feature type. In other words, we extracted each of the five feature types using the six unit forms of a text. After that, we generated the five vanilla DNN based ensemble classifiers corresponding to the feature types and then we measured the accuracy-based performance for the development data. As shown in the second, third, fourth, fifth, and sixth rows of Table 2 , the accuracies were ranged from 0.8273 to 0.8364 for each classifier using the $S V M_{\text {output }}^{\text {count }}$, 


\begin{tabular}{lclc}
\hline Feature & $\begin{array}{l}\text { Feature } \\
\text { dimension }\end{array}$ & $\begin{array}{l}\text { Norm. } \\
\text { method }\end{array}$ & Accuracy \\
\hline Official baseline & & 0.7236 \\
\hline \multicolumn{4}{l}{ Late fusion: vanilla DNN based ensemble classifier } \\
\hline (a) $S V M_{\text {output }}^{\text {count }}$ & 66 & Mean/Var. & 0.8345 \\
(b) $S V M_{\text {output }}^{L S A 200 / L D A 10}$ & 66 & Mean/Var. & 0.8364 \\
(c) $D N N_{\text {output }}^{\text {SA2000/LDA10 }}$ & 66 & Mean/Var. & 0.8345 \\
(d) $D N N_{\text {bottleneck }}^{L S A 200 / L D 10}$ & 192 & Mean/Var. & 0.8273 \\
(e) $R a w^{L S A 2000 / L D A 10}$ & 60 & Mean/Var. & 0.8318 \\
(a)+(b)+(c)+(d)+(e) (ETRI-SLP) & 450 & Mean/Var. & 0.8445 \\
\hline
\end{tabular}

Table 2: Performance comparison of the proposed method for the essay track when evaluating the development set, where 'Mean/Var.' indicates the normalization to the zero mean and unit variance.

$$
\begin{aligned}
& S V M_{\text {output }}^{L S A 2000 / L D A 10}, \quad D N N_{\text {output }}^{L S A 2000 / L D A 10}, \\
& D N N_{\text {bottleneck }}^{L S A 2000 / L D A 10}, \text { and } R a w^{L S A 2000 / L D A 10},
\end{aligned}
$$
respectively. Thus, it could be noted that each feature type successes to combine the six unit forms.

Finally, the accuracy of the proposed ETRI-SPL NLI for the essay track was 0.8445 using the thirty features by combining the six unit forms and the five feature types, as shown in the last row of the figure. When compared to the above rows of the figure, we concluded that the thirty features were well combined for the NLI.

\subsection{The experimental setup and its performances for the speech track}

For the L1 detection of the speech track using the transcription text and i-vector feature of a speech response, we used the multi-column deep-stacking DNN based ensemble classifier with the assumption that the text-related features were clearly heterogeneous to the speech-related i-vector feature. Moreover, we empirically selected the feature, $\operatorname{Raw}^{L S A 2000 / L D A 10}$, for the efficient combination with the text-related features and the i-vector feature. The submitted ETRI-SPL NLI for the speech track was performed as follows.

We first transformed each transcription text into the six unit forms and then extracted the $R a w^{L S A 2000 / L D A 10}$ for each unit-transformed text. In addition, we used the 800-dimensional i-vector feature for each speech response signal. The text-related feature was then normalized to a zero mean and unit variance and the i-vector feature was normalized using a LDA normalization. As shown in Fig. 4(b), the $\operatorname{Raw}^{L S A 2000 / L D A 10}$ and i-vector features were fed into the LSA/LDA feature layers and the i-vector layers, respectively. The node values of the last hidden layer of each

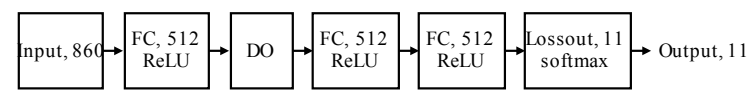

(a) Late fusion: vanilla DNN based ensemble classifier

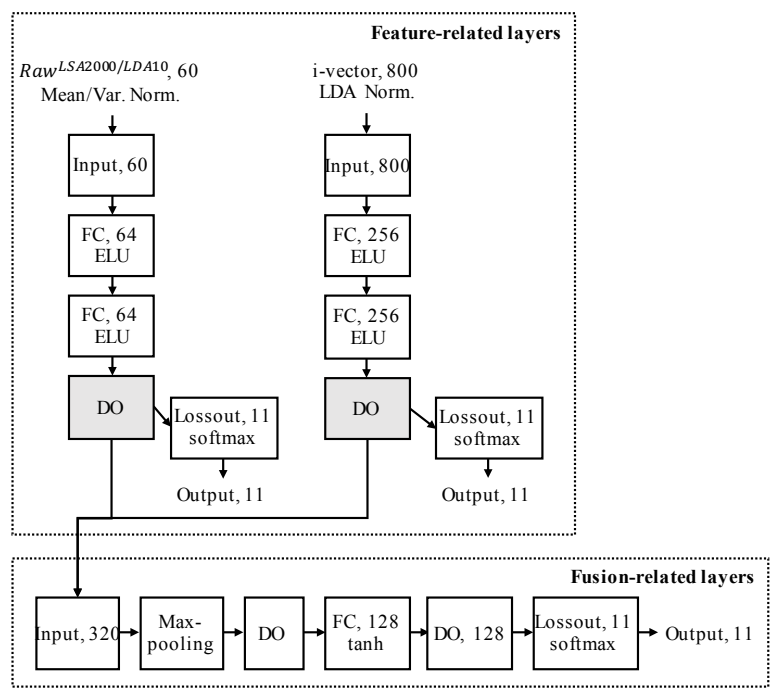

(b) Early fusion: multi-column deep-stacking DNN based ensemble classifier

Figure 4: Configuration of the two DNN based ensemble classifiers for the speech track, where each block indicates a layer of the DNN and the number in a block indicates the number of nodes in the corresponding layer.

feature layers were then connected to the input layer of the fusion layers. Each feature layers consisted of an input layer, the first and second FC hidden layers, the third DO hidden layer, and an output layer, where the FC layers contained 64 and 256 nodes for $\operatorname{Raw}^{L S A 2000 / L D A 10}$ and i-vector, respectively, with an exponential linear unit (ELU) activation function. And, the fusion layers consisted of an input layer, the first max-pooling hidden layer, the second and fourth DO hidden layers, the third FC hidden layer, and an output layer, where the FC layer contained 256 nodes with a tanh activation function.

Prior to the performance evaluation of the proposed ETRI-SPL NLI for the speech track, we evaluated the performance corresponding to each text-related feature, the i-vector feature, and the feature combinations using a vanilla DNN based ensemble classifier, as shown in Fig. 4(a). To this end, the extracted features were concatenated into one feature and then the concatenated feature was normalized using an LDA normalization since the i-vector feature was well matched with the LDA normalization rather than a normalization to a zero mean and unit variance. After that, the normalized feature was fed into the input layer of the vanilla 


\begin{tabular}{|c|c|c|c|}
\hline Feature & $\begin{array}{l}\text { Feature } \\
\text { dim. }\end{array}$ & $\begin{array}{l}\text { Norm. } \\
\text { method }\end{array}$ & Accuracy \\
\hline \multicolumn{3}{|l|}{ Official baseline with transcription } & 0.5200 \\
\hline \multicolumn{3}{|l|}{ Official baseline with i-vector } & 0.7400 \\
\hline \multicolumn{3}{|c|}{ Official baseline with transcription \& i-vector } & 0.7573 \\
\hline \multicolumn{4}{|c|}{ Early or Late fusion: vanilla DNN based ensemble classifier } \\
\hline (a) $S V M_{\text {output }}^{\text {count }}$ & 66 & LDA & 0.4545 \\
\hline (b) $S V M_{\text {output }}^{L S A 2000 / L D A 10}$ & 66 & LDA & 0.5827 \\
\hline (c) $D N N_{\text {output }}^{L S A 2000 / L D A 10}$ & 66 & LDA & 0.5782 \\
\hline (d) $D N N_{\text {bottleneck }}^{L S A 2000 / L D A 10}$ & 192 & LDA & 0.5764 \\
\hline (e) $R a w^{L S A 2000 / L D A 10}$ & 60 & LDA & 0.5836 \\
\hline (f) i-vector & 800 & LDA & 0.8082 \\
\hline (a)+(f) late fusion & 866 & LDA & 0.5118 \\
\hline (b)+(f) late fusion & 866 & LDA & 0.8245 \\
\hline (c)+(f) late fusion & 866 & LDA & 0.6682 \\
\hline (d)+(f) early fusion & 992 & LDA & 0.7345 \\
\hline$\underline{\text { (e)+(f) early fusion }}$ & 860 & LDA & 0.8309 \\
\hline (b)+(c)+(f) late fusion & 932 & LDA & 0.6627 \\
\hline (b)+(d)+(f) late fusion & 1058 & LDA & 0.7127 \\
\hline (b)+(e)+(f) late fusion & 926 & LDA & 0.8145 \\
\hline (c) $+(\mathrm{d})+(\mathrm{f})$ late fusion & 1058 & LDA & 0.6655 \\
\hline$(\mathrm{c})+(\mathrm{e})+(\mathrm{f})$ late fusion & 926 & LDA & 0.6609 \\
\hline (d)+(e)+(f) early fusion & 1052 & LDA & 0.7155 \\
\hline (b) $+(\mathrm{c})+(\mathrm{d})+(\mathrm{f})$ late fusion & 1124 & LDA & 0.6673 \\
\hline (b) $+(\mathrm{c})+(\mathrm{e})+(\mathrm{f})$ late fusion & 992 & LDA & 0.6627 \\
\hline (b) $+(d)+(e)+(f)$ late fusion & 1118 & LDA & 0.7155 \\
\hline$(\mathrm{c})+(\mathrm{d})+(\mathrm{e})+(\mathrm{f})$ late fusion & 1118 & LDA & 0.6609 \\
\hline$(\mathrm{b})+(\mathrm{c})+(\mathrm{d})+(\mathrm{e})+(\mathrm{f})$ late fusion & 1184 & LDA & 0.6582 \\
\hline \multicolumn{4}{|c|}{ Early fusion: multi-column deep-stacking DNN based ensemble classifier } \\
\hline (a)+(f) & 866 & Mean/Var./LDA & 0.8109 \\
\hline (b) $+(\mathrm{f})$ & 866 & Mean/Var./LDA & 0.8527 \\
\hline$\overline{(c)+(f)}$ & 866 & Mean/Var./LDA & 0.8473 \\
\hline (d) $+(\mathrm{f})$ & 992 & Mean/Var./LDA & 0.8491 \\
\hline (e)+(f) & 860 & Mean/Var./LDA & 0.8591 \\
\hline$(d)+(e)+(f)$ & 1052 & Mean/Var./LDA & 0.8455 \\
\hline$(d)^{\prime}+(e)+(f)($ ETRI-SLP) & 1052 & Mean/Var./LDA & 0.8545 \\
\hline
\end{tabular}

Table 3: Performance comparison of the proposed method for the speech track when evaluating the development data, where the underlined and the bolded represent the remarkable system and the submitted system, respectively. The 'early fusion' of the vanilla DNN based ensemble classifier indicates a classifier that uses no feature-based classifier. And, 'Mean/Var.' indicates a normalization to a zero mean and unit variance. The (d)' means the noisy data of the $D N N_{\text {bottleneck }}^{L S A 2000 / L D A 10}$, which was an unexpected data.

DNN based ensemble classifier. The vanilla DNN based ensemble classifier consisted of an input layer, the first, third, and fourth FC hidden layers, the second DO hidden layer, and an output layer, where each FC hidden layer contained 512 nodes with a rectified linear unit (RELU) activation function. Also, it was noted that the number of nodes of the FC hidden layer was increased according to the increase of the dimension of the input feature data.

From the fourth row to the ninth row of Table 3 , it was noted that the i-vector feature outperformed the text-related features. Among the text- related features, the LSA-LDA based features had better performances when compared to the countbased feature. From the tenth row to the fourteenth row of the table, the $S V M_{\text {output }}^{L S A 200 / L D A 10}$ and $R a w^{L S A 2000 / L D A 10}$ improved the only i-vector feature when combining one text-related feature and the i-vector feature. However, it was shown from the fifteenth row to the twenty-fifth row of the table that the combination with two or more text-related features and the i-vector feature did not improve the combination with one text-related feature and the i-vector feature. It was summarized that there was no improvement on the combination with two or more text-related features since the text-related features had similar information using the same unit forms.

From the twenty-sixth row to the thirtieth row of the table, all the combinations of one text-related feature and the i-vector feature were improved using the multi-column deep-stacking DNN based ensemble classifier when compared to the use of one feature; only two features were improved using the vanilla DNN based ensemble classifier. Moreover, the thirty-first row of the table showed that the performance of the combination with the two text-related features and ivector feature was slightly degraded; however, the degree of the performance degradation was marginal. Finally, the last row of the table presented the performance of the submitted system. In fact, the original intention was to combine the two text-related features and i-vector feature. Unfortunately, we found that the noisy data was inserted as the $D N N_{\text {bottleneck }}^{L S A 200 / L D A 10}$ after the submission. However, from the performance evaluation, we could examine that the multi-column deep-stacking DNN based ensemble classifier had the robust performance to a noisy data.

\subsection{The experimental setup and its performances for the fusion track}

For the L1 detection of the fusion track using the text of an essay response and the transcription text and i-vector feature of a speech response, we used the multi-column deep-stacking DNN based ensemble classifier. For the efficient combination with the text-related features and the speech i-vector feature, we empirically selected the $R a w^{L S A 2000 / L D A 10}$ among nonclassifier-based features. The submitted ETRISPL NLI for the fusion track was performed as 


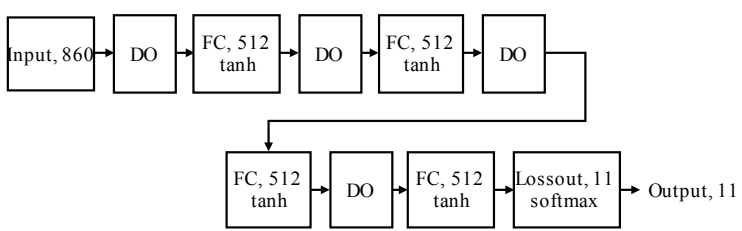

(a) Late fusion: vanilla DNN based ensemble classifier

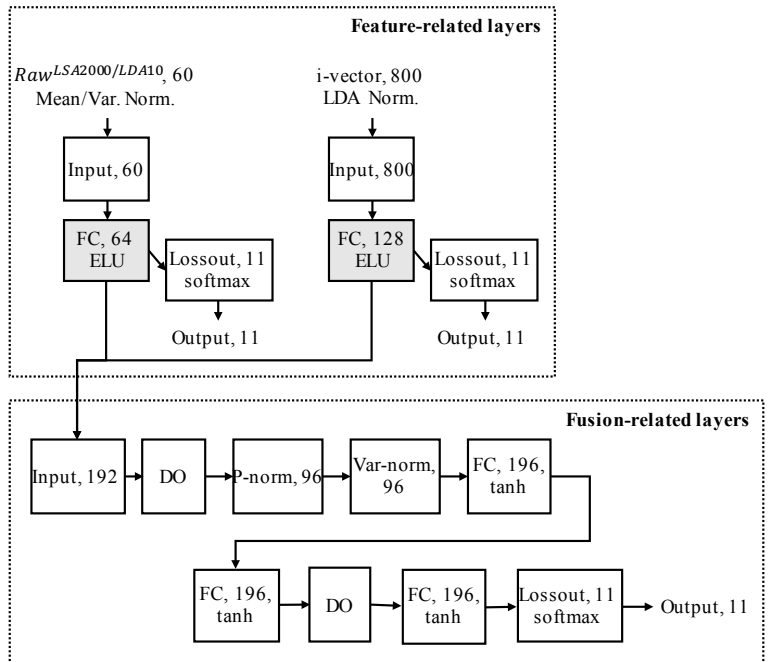

(b) Early fusion: multi-column deep-stacking DNN based ensemble classifier

Figure 5: Configuration of the two DNN based ensemble classifiers for the fusion track, where each block indicates a layer of a DNN and the number in a block indicates the number of nodes in the layer corresponding to a block.

follows.

We first transformed each text of the essay and speech transcription into the six unit forms and then extracted the Raw ${ }^{L S A 2000 / L D A 10}$ for each unit-transformed text. To fuse an essay response and a speech response, the count-based vector of the speech transcription text was appended to the count-based vector of the essay text for each pair of an essay text and speech transcription text during the feature extraction of the Raw ${ }^{L S A 2000 / L D A 10}$. We also used the 800dimensional i-vector feature of each speech response signal. The text-related features were then normalized to a zero mean and unit variance and the i-vector feature were normalized using a LDA normalization. As shown in Fig. 5(b), the $R a w^{L S A 2000 / L D A 10}$ and i-vector features were fed into the LSA/LDA feature layers and the ivector layers, respectively. The node values of the last hidden layer of each feature layers were then connected to the input layer of the fusion layers. Each feature layers consisted of an input layer, the FC hidden layer, and an output layer,

\begin{tabular}{|c|c|c|c|}
\hline $\begin{array}{l}\text { Feature } \\
\text { type }\end{array}$ & $\begin{array}{l}\text { Feature } \\
\text { dim. }\end{array}$ & $\begin{array}{l}\text { Normalization } \\
\text { method }\end{array}$ & Accuracy \\
\hline Official baseline & & & 0.7836 \\
\hline \multicolumn{4}{|c|}{ Late fusion: vanilla DNN based ensemble classifier } \\
\hline (a) $S V M_{\text {output }}^{\text {count }}$ & 66 & LDA & 0.6309 \\
\hline (b) $S V M_{\text {output }}^{L S A 2000 / L D A 10}$ & 66 & LDA & 0.8309 \\
\hline (c) $D N N_{\text {output }}^{L S A 2000 / L D A 10}$ & 66 & LDA & 0.8518 \\
\hline (d) $D N N_{\text {bottleneck }}^{L S A 2000 / L D A 10}$ & 192 & LDA & 0.8418 \\
\hline (e) $R a w^{L S A 2000 / L D A 10}$ & 60 & LDA & 0.8291 \\
\hline (f) i-vectors & 800 & LDA & 0.7900 \\
\hline (g) $S V M_{\text {output }}^{\text {count }}$ & 66 & Mean/Var. & 0.8582 \\
\hline (h) $S V M_{\text {output }}^{L S A 2000 / L D A 10}$ & 66 & Mean/Var. & 0.8482 \\
\hline (i) $D N N_{\text {output }}^{L S A 2000 / L D A 10}$ & 66 & Mean/Var. & 0.8400 \\
\hline (j) $D N N_{\text {bottleneck }}^{L S A 2000 / L D A 10}$ & 192 & Mean/Var. & 0.8400 \\
\hline (k) $R a w^{L S A 2000 / L D A 10}$ & 60 & Mean/Var. & 0.8473 \\
\hline (l) i-vectors & 800 & Mean/Var. & 0.5864 \\
\hline (e)+(f) late fusion & 860 & LDA & 0.9155 \\
\hline \multicolumn{4}{|c|}{ Early fusion: multi-column deep-stacking DNN based ensemble classifie } \\
\hline (e)+(f) (ETRI-SLP) & 860 & Mean/Var./LDA & 0.9164 \\
\hline
\end{tabular}

Table 4: Performance comparison of the proposed method for the fusion track when evaluating the development data, where the bolded represent the submitted system. 'Mean/Var.' indicates a normalization to a zero mean and unit variance.

where the FC layers contained 64 and 128 nodes for $R a w^{L S A 2000 / L D A 10}$ and i-vector, respectively, with an ELU activation function. And, the fusion layers consisted of an input layer, the first and sixth DO hidden layers, the fourth, fifth, and seventh FC hidden layers, the second p-norm pooling hidden layer, the third variance normalization hidden layer, and an output layer, where each FC layer contained 196 nodes with a tanh activation function and the p-norm and variance normalization layers contained 96 nodes.

Prior to the performance evaluation of the proposed ETRI-SPL NLI for the fusion track, we evaluated the performance corresponding to the $R a w^{L S A 2000 / L D A 10}$, the i-vector feature, and the feature combination, respectively, using a vanilla DNN based ensemble classifier, as shown in Fig. 5(a). To this end, the extracted features were concatenated into one feature and then the concatenated feature was normalized using an LDA normalization. The normalized feature was then fed into the input layer of the vanilla DNN based ensemble classifier. The vanilla DNN based ensemble classifier consisted of an input layer, the first, third, fifth, and seventh DO hidden layers, the second, fourth, sixth, and eighth FC hidden layers, and an output layer, where each FC hidden layer contained 512 nodes with a tanh activation function. 
It was shown from the second row to the seventh row of Table 4 that the LSA-LDA features had better performances than the count-based feature and i-vector feature when applying an LDA normalization. It was shown from the eighth row to the thirteenth row of the table that the count-based feature and i-vector feature were well matched with a normalization to a zero mean and unit variance and with an LDA normalization, respectively. From the fourteenth and fifteenth rows of the table, the two DNN based ensemble classifiers obtained the similar accuracies when using the same feature combination. It was noted from the experiments that the multi-column deep-stacking DNN based ensemble classifier worked better than the vanilla DNN based ensemble classifier when the features were heterogeneous and the performance differences were significant.

\section{Performance of the test data set and discussions}

This section first reports the official performance comparison based on the F1 (macro) score for the 1,100 test data set. Moreover, we present the official ranks that are grouped by a McNemar's test. Thus, we regard that the a same group has a comparable performance. After that, we conclude with our findings and discussions.

Table 5 and Fig. 6 present the performance comparisons and the confusion matrices of the submitted ETRI-SPL NLI systems for the essay track, the speech track, and the fusion track, respectively. For the essay track, the F1 (macro) scores are $0.7104,0.8601$, and 0.8818 , for the baseline system, the ETRI-SPL system, and the ItaliaNLP (top-scored) system. In other words, the proposed system has the improved performance when compared to the baseline system; however, the proposed system has a slightly lower performance when compared to the top-scored system. For the speech track, the F1 (macro) scores are $0.7980,0.8664$, and 0.8755 , for the baseline system, the ETRI-SPL system, and the UnibucKernel (top-scored) system. That is, the proposed system has the comparable performance to the top-scored system. For the fusion track, the F1 (macro) scores are $0.7901,0.9220$, and 0.9319 , for the baseline system, the ETRI-SPL system, and the UnibucKernel (top-scored) system. That is, the proposed system also has the comparable performance to the top-scored system.

\begin{tabular}{l|l|c|cc}
\hline \multirow{2}{*}{ Track } & Team & $\begin{array}{r}\text { Rank } \\
\text { group }\end{array}$ & F1 (macro) & Accuracy \\
\hline \multirow{3}{*}{ Essay } & Baseline & - & 0.7104 & 0.7109 \\
\cline { 2 - 5 } & ETRI-SLP & 2 & 0.8601 & 0.8600 \\
\cline { 2 - 5 } & ItaliaNLP (Top-scored) & 1 & 0.8818 & 0.8818 \\
\hline \multirow{3}{*}{ Speech } & Baseline & - & 0.7980 & 0.7982 \\
\cline { 2 - 5 } & ETRI-SLP & 1 & 0.8664 & 0.8664 \\
\cline { 2 - 5 } & UnibucKernel (Top-scored) & 1 & 0.8755 & 0.8755 \\
\hline \multirow{3}{*}{ Fusion } & Baseline & - & 0.7901 & 0.7909 \\
\cline { 2 - 5 } & ETRI-SLP & 1 & 0.9220 & 0.9218 \\
\cline { 2 - 5 } & UnibucKernel (Top-scored) & 1 & 0.9319 & 0.9318 \\
\hline
\end{tabular}

Table 5: Performance comparison based on the F1 and accuracy metrics of the proposed method for the essay, speech, and fusion tracks when evaluating the test data set. The first, second, third rows of each track indicate the official baseline system, the proposed system, and the top-scored system, respectively.

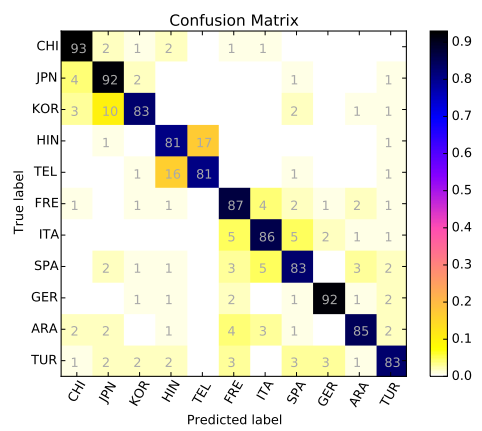

(a) Essay track

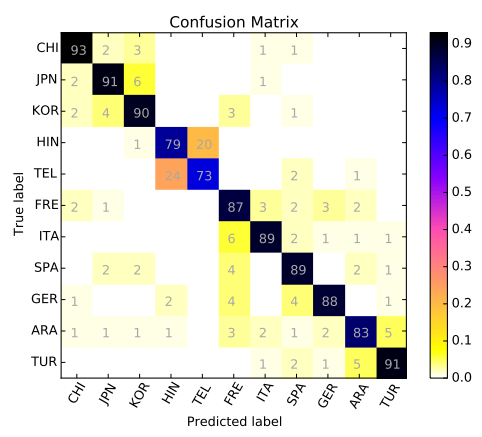

(b) Speech track

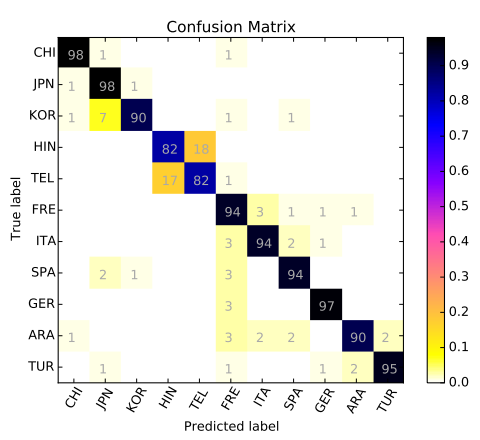

(c) Fusion track

Figure 6: Confusion matrixes of the ETRI-SLP NLI systems for the essay track, the speech track, and the fusion track, respectively, when evaluating the test data set. 
In conclusion, we proposed the deep-learning based NLI using an LSA for the NLI Shared Task 2017. To this end, we extracted the LSA features using the six unit forms of character 4/5/6-grams and word 1/2/3-grams. Especially, we used 2,000rank LSA features in order to capture the language information whereas the lower-rank LSA feature was used to the document topic-related applications. Next, the 2000-rank LSA feature was reduced into a 10-dimensional feature using LDA. It was noted from the NLI experiments that the LSA/LDA features performed well in the NLI Shared Task 2017 when compared to the countbased features, especially for the speech track.

For a fusion of the heterogeneous features such as the combination of a text-related feature and an i-vector feature, we designed two DNN based ensemble classifiers: (a) the vanilla DNN based ensemble classifier for late fusion and (b) the multicolumn deep-stacking DNN based ensemble classifier for early fusion. The vanilla DNN based ensemble classifier was a late fusion classifier that combined the independently trained featurerelated classifiers whereas the multi-column deepstacking DNN based ensemble classifier was an early fusion classifier that combined the features in one fusion network. It was shown from the NLI experiments that the two DNN based ensemble classifiers had the comparable performances when the feature type and the performance were similar to each other. On the other hand, the multi-column deep-stacking DNN based ensemble classifier had a better performance when the the feature type and the performance were significantly different.

It was shown from the experiments on the NLI Shared Task 2017 that the F1 (macro) scores were obtained as $0.8601,0.8664$, and 0.9220 , for the essay track, the speech track, and the fusion track, respectively. The performances for the speech and fusion tracks were comparable to the top-ranked systems whereas the performance for the essay track had a second-ranked performance.

Our findings from the NLI Shared Task 2017 were summarized as follows:

\section{Unit form for a text}

We used the six unit forms of character 4/5/6grams and word $1 / 2 / 3$-grams. From the tenth row to the fourteenth row of Table 3 , it was noted that the combination of the multiple text-related features had no improvement because the proposed text-related features were originated from the same unit forms. Therefore, we expected that the performance for the essay track would be improved if the additional unit forms were adopted.

2. Feature type for a text: LSA-LDA feature From the NLI experiments, it was noticed that the 2,000-rank LSA-LDA feature worked well for the NLI Shared Task 2017. Especially, LSA-LDA feature had a better performance than the count-based feature for the speech track. Moreover, the LSA-LDA feature worked well on both a normalization to a zero mean and unit variance and an LDA normalization whereas the count-based feature worked on the only normalization to a zero mean and unit variance.

\section{Normalization of an i-vector feature}

It was observed from the experiments that the $\mathrm{i}$-vector feature of a speech response signal worked well on an LDA normalization than a normalization to a zero mean and unit variance.

\section{DNN-based ensemble classifier}

We attempted to use of a state-of-the-art deep learning method for the L1 classification by designing two DNN based ensemble classifiers: (a) the vanilla DNN based ensemble classifier for late fusion and (b) the multicolumn deep-stacking DNN based ensemble classifier for early fusion. From the performance comparison of the other systems, it was seen that the proposed classifiers worked properly. Moreover, the multi-column deepstacking DNN based ensemble classifier was better when the heterogeneous features had significant performance differences. In addition, we expected that the more detailed experiments of the DNN configurations and the feature combinations would improve the performance, especially using a more large amount data (Cheng et al., 2015).

\section{Acknowledgements}

This work was partially supported by the ICT R\&D program of MSIP/IITP. [R0126-15-1117, Core technology development of the spontaneous speech dialogue processing for the language learning] and by Electronics and Telecommunications Research Institute (ETRI) grant funded by the Korean government. [17ZS1800, Development of self-improving and human-augmenting cognitive computing technology] 


\section{References}

Jerome R Bellegarda. 2000. Exploiting latent semantic information in statistical language modeling. Proc. IEEE 88(8):1279-1296. https://doi.org/10.1109/5.880084.

Jian Cheng, Xin Chen, and Angeliki Metallinou. 2015. Deep neural network acoustic models for spoken assessment applications. Speech Communication 73:14 - 27. https://doi.org/10.1016/j.specom.2015.07.006.

Euisok Chung and Jeon Gue Park. in review. Sentencechain based seq2seq model for corpus expansion. ETRI Journal .

Dan C. Ciresan, Ueli Meier, and Jürgen Schmidhuber. 2012. Multi-column deep neural networks for image classification. CoRR abs/1202.2745. http://arxiv.org/abs/1202.2745.

František Grézl, Martin Karafiát, Stanislav Kontár, and Jan Černocký. 2007. Probabilistic and bottle-neck features for lvcsr of meetings. In Proc. IEEE International Conference on Acoustics, Speech and Signal Processing (ICASSP 2007). IEEE Signal Processing Society, pages 757-760. https://doi.org/10.1109/ICASSP.2007.367023.

HyungBae Jeon and Soo-Young Lee. 2016a. Initializing deep learning based on latent dirichlet allocation for document classification. Neural Information Processing. ICONIP 2016. Lecture Notes in Computer Science 9949. https://doi.org/10.1007/978-3319-46675-0_70.

HyungBae Jeon and Soo-Young Lee. 2016b. Language model adaptation based on topic probability of latent dirichlet allocation. ETRI Journal 38(3):487-493. https://doi.org/10.4218/etrij.16.0115.0499.

T. Joachims. 1999. Making large-scale SVM learning practical. In B. Schölkopf, C. Burges, and A. Smola, editors, Advances in Kernel Methods - Support Vector Learning, MIT Press, Cambridge, MA, chapter 11, pages 169-184.

Shervin Malmasi. 2016. Native Language Identification: Explorations and Applications. Ph.D. thesis. http://hdl.handle.net/1959.14/1110919.

Shervin Malmasi and Mark Dras. 2015. Multilingual Native Language Identification. In Natural Language Engineering. https://doi.org/10.1017/S1351324915000406.

Shervin Malmasi, Keelan Evanini, Aoife Cahill, Joel Tetreault, Robert Pugh, Christopher Hamill, Diane Napolitano, and Yao Qian. 2017. A Report on the 2017 Native Language Identification Shared Task. In Proceedings of the 12th Workshop on Building Educational Applications Using NLP. Association for Computational Linguistics, Copenhagen, Denmark.
Shervin Malmasi, Marcos Zampieri, Nikola Ljubešić, Preslav Nakov, Ahmed Ali, and Jörg Tiedemann. 2016. Discriminating between Similar Languages and Arabic Dialect Identification: A Report on the Third DSL Shared Task. In Proceedings of the VarDial Workshop. Osaka, Japan.

Björn Schuller, Stefan Steidl, Anton Batliner, Julia Hirschberg, Judee K. Burgoon, Alice Baird, Aaron Elkins, Yue Zhang, Eduardo Coutinho, and Keelan Evanini. 2016. The INTERSPEECH 2016 Computational Paralinguistics Challenge: Deception, Sincerity \& Native Language. In Interspeech 2016. pages 2001-2005. https://doi.org/10.21437/Interspeech.2016-129.

C. G. M. Snoek, M. Worring, and A. W. M. Smeulders. 2005. Early versus late fusion in semantic video analysis. In ACM International Conference on Multimedia. pages 399?-402. https://doi.org/10.1145/1101149.1101236.

Joel Tetreault, Daniel Blanchard, and Aoife Cahill. 2013. A Report on the First Native Language Identification Shared Task. In Proceedings of the Eighth Workshop on Building Educational Applications Using NLP. Association for Computational Linguistics, Atlanta, GA, USA.

Marcos Zampieri, Shervin Malmasi, Nikola Ljubešić, Preslav Nakov, Ahmed Ali, Jörg Tiedemann, Yves Scherrer, and Noëmi Aepli. 2017. Findings of the VarDial Evaluation Campaign 2017. In Proceedings of the Fourth Workshop on NLP for Similar Languages, Varieties and Dialects (VarDial). Valencia, Spain, pages 1-15. 